\title{
Choice of recipient vessels in muscle-sparing transverse rectus abdominis myocutaneous flap breast reconstruction: A comparative study
}

\author{
Kyung-Chul Moon, Jae-Min Lee, Si-Ook Baek, Seo-Yoon Jang, Eul-Sik Yoon, Byung-Il Lee, \\ Seung-Ha Park \\ Department of Plastic Surgery, Korea University College of Medicine, Seoul, Korea
}

Background Thoracodorsal vessels (TDVs) and internal mammary vessels (IMVs) have both been widely employed as recipient vessels for use in free muscle-sparing transverse rectus abdominis myocutaneous (MS-TRAM) flaps. However, whether TDVs or IMVs are preferable as recipient vessels for autologous breast reconstruction with a free MS-TRAM flap remains controversial. The purpose of this study was to compare the clinical outcomes when TDVs were used as recipient vessels to those obtained when IMVs were used as recipient vessels for autologous breast reconstruction with a free MS-TRAM flap.

Methods A retrospective matched-cohort study was performed. We retrospectively reviewed data collected from patients who underwent a free MS-TRAM flap for autologous breast reconstructions after mastectomy between March 2003 and June 2013. After a one-to-one matching using age, 100 autologous breast reconstructions were selected in this study. Of the 100 breast reconstructions, 50 flaps were anastomosed to TDVs and 50 to IMVs. Patient demographics and clinical outcomes including operation time, length of hospital stay, postoperative complications, and aesthetic score were compared between the two groups.

Results No statistically significant differences were found between the two groups in patient demographics and clinical outcomes, including the complication rates and aesthetic scores. There were no major complications such as total or partial flap loss in either group.

Conclusions The results of our study demonstrate that both TDVs and IMVs were safe and efficient as recipient vessels in terms of the complication rates and aesthetic outcomes.

Keywords Mammary arteries / Postoperative complications / Reconstructive surgical procedures

Received: 29 Jul $2018 \bullet$ Revised: 8 Oct $2018 \bullet$ Accepted: 30 Oct 2018

pISSN: 2234-6163・ elSSN: 2234-6171 • https://doi.org/10.5999/aps.2018.00913• Arch Plast Surg 2019;46:140-146
Correspondence: Eul-Sik Yoon Department of Plastic Surgery, Korea University College of Medicine, 73 Inchon-ro, Seongbuk-gu, Seoul 02841, Korea

Tel: $+82-2-920-5440$

Fax: +82-2-922-7437

E-mail: yesanam2@korea.ac.kr

\section{INTRODUCTION}

Thoracodorsal vessels (TDVs) and the internal mammary vessels (IMVs) are the two main recipient vessels for free musclesparing transverse rectus abdominis myocutaneous (MS-
TRAM) flaps. Traditionally, TDVs have been used as recipient vessels when they are partially exposed after axillary lymph node dissection during mastectomy, as they have consistent anatomy $[1,2]$. Furthermore, the dissection of TDVs affords a small but significant opportunity to detect occult regional me- 
tastasis [3]. In addition, TDVs do not increase the risk of lymphedema [1] compared to other recipient vessels, and they are not adversely affected by prior radiotherapy [2]. Despite these advantages of TDVs, lateral fullness after breast reconstructions is their main drawback.

To overcome these drawbacks, recent studies have shown that IMVs may be preferable recipient vessels for autologous breast reconstruction. IMVs may provide a better match in terms of caliber to the deep inferior epigastric vessels (DIEVs) and are more accessible than TDVs during autologous breast reconstruction procedures. In addition, using IMVs as the recipient vessels may make it possible to position the flap more medially. Furthermore, the use of IMVs decreases of the risk of lymphedema and nerve and vessel injuries in the axillary area. However, IMVs also have several disadvantages, including greater postoperative chest pain, iatrogenic pneumothorax, and chest contour deformity because they require excision of costal cartilage [4].

Despite the debates on recipient vessels, it has been reported that both TDVs and IMVs provide comparable rates of overall flap survival and vessel conversion [5]. Although multiple comparative studies have described recipient vessels for autologous breast reconstruction, an insufficient number of studies have compared complication rates and aesthetic outcomes between the use of TDVs and IMVs as recipient vessels. Furthermore, to our knowledge, no reports have evaluated and compared these two categories of recipient vessels using a one-to-one matching analysis. Thus, the purpose of this study was to compare clinical outcomes, such as the complication rate and aesthetic outcomes, between these two vessel types in MS-TRAM flaps using a one-to-one matching analysis. As autologous breast reconstruction becomes increasingly popular among Koreans, it is important that trainees become skilled in the required techniques; therefore, we prepared visual materials comparing the two types of recipient vessels. It is our hope that this article will provide a basis for understanding the possible recipient vessels of MS-TRAM flaps and advancing the surgical skills of trainee at departments of plastic surgery.

\section{METHODS}

This retrospective study received approval from Institutional Review Board of Korea University Guro Hospital, and all patients provided written informed consent. After a one-to-one matching using age, an analysis of 100 consecutive breast reconstructions was conducted. For these patients, free muscle sparing (MS) TRAM flaps were harvested using DIEVs as donor vessels by a single surgeon (ESY) between March 2003 and June 2013. Overall, TDVs were used as recipient vessels in 50 breast reconstructions while IMVs were used as recipient vessels in 50 breast reconstructions. These 100 reconstructions were all immediate reconstructions after mastectomy. The mean followup period was $62.3 \pm 47.1$ weeks (range, $10-142$ weeks).

\section{Operative technique}

Under loupe magnification, the recipient vessels were exposed prior to anastomosis of the flaps in all patients. TDVs were used for recipient vessels when axillary dissection was performed before autologous breast reconstruction. The TDVs were exposed and dissected to be free from surrounding soft tissues. After flap elevation, end-to-end anastomoses were performed, using either interrupted 9-0 nylon sutures for both the artery and vein or sutures for the artery and a coupler for the vein (Fig. 1, Supple-

\section{Fig. 1. Anastomosis of donor and recipient vessels}

(A) Intraoperative photograph after anastomosis of a thoracodorsal vessel. (B) Intraoperative photograph after anastomosis of an internal mammary vessel.
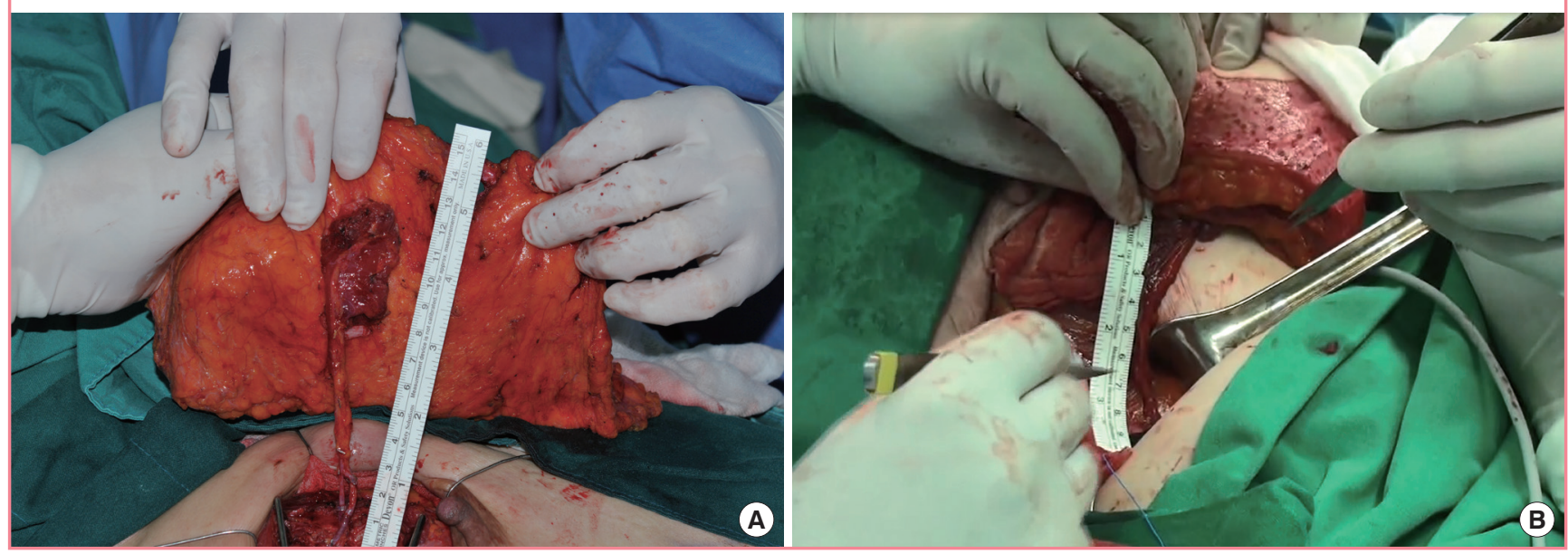
mental Video 1).

The IMVs were approached by splitting the pectoralis major muscle overlying the third costal cartilage. The perichondrium was incised along the mid-anterior surface from the junction of the sternum to $5 \mathrm{~cm}$ medial to the costochondral junction. The cartilage was separated from the anterior to the posterior surface. After the upper and lower edges of the costal cartilage were separated from the perichondrium, a sharp blade and a rongeur were used to remove the cartilage to provide more space for anastomosis without causing pneumothorax. Once fully dissected, IMVs were divided at the intercostal space. End-to-end anastomoses were then performed using either interrupted 9-0 nylon sutures for both the artery and vein or sutures for the artery and a coupler for the vein (Fig. 1, Supplemental Video 2).

\section{Evaluation}

The following data were reviewed: recipient vessels; mastectomy type; breast cancer stage; operation time; length of hospital stay; contralateral breast surgery; medical history; including age, body mass index, smoking history, and history of preoperative chemotherapy; and complications. Complications were subdivided into major complications (i.e., total or partial flap loss, flap congestion, deep vein thrombosis, fat embolism, and pneumothorax) and minor complications (i.e., wound dehiscence, skin necrosis, hematoma, and seroma). At the last follow-up visit, the aesthetic outcome was assessed by the patient, four blinded nonmedical observers, and two blinded plastic surgeons using a 4-point linear analogue scale: poor, 1; fair, 2; good, 3; excellent, 4. The self-assessment by patients was made based on the Global Aesthetic Improvement Scale, and the objective assessment by other observers was made using four parameters: breast volume, symmetry, position of the inframammary fold, and scar

\section{Table 1. Patient demographics and outcomes}

\begin{tabular}{|lccc|}
\hline Variable & TDV $(\mathbf{n}=\mathbf{5 0})$ & IMV $(\mathbf{n}=\mathbf{5 0})$ & P-value \\
\hline Age (yr) & $45.33 \pm 8.52$ & $46.58 \pm 7.12$ & 0.985 \\
Operation time (hr) & $10.08 \pm 1.39$ & $10.21 \pm 1.68$ & 0.524 \\
Hospital stay (day) & $20.19 \pm 4.17$ & $21.23 \pm 6.72$ & 0.130 \\
Aesthetic score & $22.13 \pm 5.76$ & $23.05 \pm 5.14$ & 0.769 \\
Complication & $13(26)$ & $15(30)$ & 0.528 \\
Contralateral breast surgery & $5(10)$ & $8(16)$ & 0.391 \\
BMI > 25 kg/m² & $11(22)$ & $9(18)$ & 0.482 \\
Smoking & $4(8)$ & $3(6)$ & 0.837 \\
Chemotherapy & $5(10)$ & $3(6)$ & 0.471 \\
Hypertension & $3(6)$ & $3(6)$ & 0.698 \\
Diabetes mellitus & $2(4)$ & 0 & 0.244 \\
Cardiologic disease & $2(4)$ & 0 & 0.244 \\
Endocrine disease & $2(4)$ & $1(2)$ & 0.141 \\
\hline Values are presented as mean \pm SD or number (\%). \\
TDV, thoracodorsal vessel; IMV, internal mammary vessel; BMl, body mass index. \\
\hline
\end{tabular}

quality. The aesthetic scores assigned by these seven evaluators were added to obtain the final aesthetic score, ranging from 7 to 28. Based on the final aesthetic score, results were categorized as poor (score of 7-11), fair (score of 12-17), good (score of 1823), and excellent (score of 24-28).

\section{Statistical analyses}

Descriptive statistics were presented as mean \pm standard deviation unless otherwise specified. After matching, a pairwise comparison of the two groups' baseline characteristics was performed using the Wilcoxon signed-rank test or the independent sample t-test to compare quantitative variables between the two groups. The Pearson chi-square test or the Fisher exact test was used to compare the complication rate and aesthetic outcomes. P-values $<0.05$ were considered to indicate statistical significance. SPSS version 20 (IBM Corp., Armonk, NY, USA) was used for all statistical analyses.

\section{RESULTS}

The patient demographics and surgical outcomes, including the mean operation time, length of hospital stay, complication rates, and aesthetic outcomes, of the TDV and IMV groups are shown in Table 1 . No statistically significant differences were found between the two groups for any variable. The most commonly performed mastectomy type and breast cancer stage were modified radical mastectomy (62\%) and stage IIa (44\%) in the TDV

Table 2. Comparison of mastectomy type and breast cancer stage between TDVs and IMVs

\begin{tabular}{|c|c|c|c|c|}
\hline Mastectomy & $\begin{array}{c}\text { TDV } \\
(n=50)\end{array}$ & $\begin{array}{c}\text { IMV } \\
(n=50)\end{array}$ & P-value & $\begin{array}{c}\text { Total } \\
(n=100)\end{array}$ \\
\hline \multicolumn{5}{|l|}{ Mastectomy type } \\
\hline MRM & 31 (62) & $23(46)$ & 0.687 & $54(54)$ \\
\hline SSM & $17(34)$ & $24(48)$ & 0.126 & $41(41)$ \\
\hline NSM & $2(4)$ & $3(6)$ & 0.532 & $5(5)$ \\
\hline \multicolumn{5}{|c|}{ Breast cancer stage } \\
\hline 0 & 0 & $20(40)$ & $<0.001^{\mathrm{b})}$ & $25(25)$ \\
\hline la & $3(6)$ & $8(16)$ & 0.312 & $15(15)$ \\
\hline $\mathrm{lb}$ & $2(4)$ & $1(2)$ & 0.341 & $1(1)$ \\
\hline Ila & $22(44)$ & $14(28)$ & 0.621 & $33(33)$ \\
\hline$\| \mathrm{lb}$ & $5(10)$ & $2(4)$ & 0.214 & $3(3)$ \\
\hline Illa & $5(10)$ & $2(4)$ & 0.214 & $7(7)$ \\
\hline Illb & $2(6)$ & 0 & 0.244 & $3(3)$ \\
\hline IIIc & $9(18)$ & 0 & $<0.001^{\text {b) }}$ & $9(9)$ \\
\hline IV & $1(2)$ & 0 & 0.352 & $1(1)$ \\
\hline Non-cancera) & 0 & $3(6)$ & $0.034^{\text {b) }}$ & $3(3)$ \\
\hline \multicolumn{5}{|c|}{ 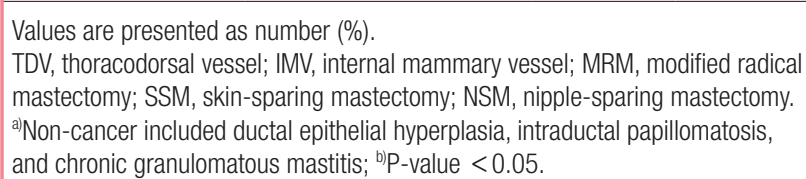 } \\
\hline
\end{tabular}


Table 3. Complication rates for TDVs and IMV

\begin{tabular}{|lcccc|}
\hline Complications & $\begin{array}{c}\text { TDV } \\
(\mathbf{n}=\mathbf{5 0})\end{array}$ & $\begin{array}{c}\text { IMV } \\
(\mathbf{n}=\mathbf{5 0})\end{array}$ & P-value & $\begin{array}{c}\text { Total } \\
(\mathbf{n}=\mathbf{1 0 0})\end{array}$ \\
\hline Major complications & 0 & 0 & - & 0 \\
$\quad$ Total flap loss & 0 & 0 & - & 0 \\
Partial flap loss & 0 & 0 & - & 0 \\
Fat embolism & 0 & 0 & - & 0 \\
Nerve injury & 0 & 0 & - & 0 \\
Pneumothorax & 0 & 0 & - & 0 \\
Minor complications & $13(26)$ & $15(30)$ & 0.628 & $28(28)$ \\
Wound dehiscence & $3(6)$ & $8(16)$ & 0.312 & $11(11)$ \\
Mastectomy skin necrosis & $7(14)$ & $3(6)$ & 0.341 & $10(10)$ \\
Hematoma & $2(4)$ & $3(6)$ & 0.412 & $5(5)$ \\
Seroma & $1(2)$ & $1(2)$ & 0.698 & $2(2)$ \\
\hline \multicolumn{4}{l}{ Values are presented as number (\%). } \\
TDV, thoracodorsal vessel; IMV, internal mammary vessel. & \\
\hline
\end{tabular}

group, respectively, and skin-sparing mastectomy (48\%) and stage O (40\%) in the IMV group, respectively (Table 2$)$. The complication rates and aesthetic outcomes for both groups are shown in Tables 3 and 4, respectively. There were no major complications such as total or partial flap loss, fat embolism, nerve injury, and pneumothorax in either group. In addition, there were no statistically significant differences in the rates of any minor complications between the two groups. Furthermore, 28 of the 50 patients (56\%) in the TDV group and 26 of the 50 patients (52\%) in the IMV group had excellent scores for aesthetic outcomes, and there were no statistically significant differences in aesthetic outcomes between the groups.

\section{DISCUSSION}

The selection of a recipient vessel is of vital importance because it not only influences flap survival and operation time, but also affects postoperative complications and aesthetic outcomes. Although the appropriate selection of recipient vessels is one of the most important factors for successful autologous breast reconstruction, the choice of a recipient vessel is largely made by reconstructive surgeons, and reflecting their comfort level, experience, flow characteristics, computed tomography angiography, and patient comorbidities. Therefore, we conducted a one-toone matched analysis to identify whether there were any significant differences in clinical outcomes between the two recipient vessels used for autologous breast reconstruction procedures.

Reconstructive surgeons may choose TDVs as recipient vessels when these vessels are thoroughly exposed following axillary node dissection [6]. However, it is necessary to harvest a flap with a long pedicle in order to insert a cosmetically appropriate flap. If TDVs are used as the recipient vessels, surgeons may encounter limitations in increasing the medial volume of
Table 4. Aesthetic outcomes for TDVs and IMVs

\begin{tabular}{|lcccc|}
\hline $\begin{array}{l}\text { Aesthetic } \\
\text { outcome }\end{array}$ & $\begin{array}{c}\text { TDV } \\
(\mathbf{n}=\mathbf{5 0})\end{array}$ & $\begin{array}{c}\text { IMV } \\
(\mathbf{n}=\mathbf{5 0})\end{array}$ & P-value & $\begin{array}{c}\text { Total } \\
(\mathbf{n}=\mathbf{1 0 0})\end{array}$ \\
\hline Excellent & $28(56)$ & $26(52)$ & 0.872 & $54(54)$ \\
Good & $12(24)$ & $18(36)$ & 0.312 & $30(30)$ \\
Fair & $8(16)$ & $6(12)$ & 0.519 & $14(14)$ \\
Poor & $2(4)$ & 0 & - & $2(2)$ \\
\hline $\begin{array}{l}\text { Values are presented as number (\%). } \\
\text { TDV, thoracodorsal vessel; IMV, internal mammary vessel. }\end{array}$ \\
\hline
\end{tabular}

the breasts, reconstructing breasts with ptosis, and maintaining the symmetry of breasts. In addition, there may be difficulties in vessel dissection due to scar formation or fibrosis of the axillary area in delayed breast reconstructions [7].

IMVs have been preferred in recent years because they can provide a better caliber match to DIEVs and they are more accessible for anastomosis than TDVs. In addition, the use of IMVs allows flaps to be positioned more medially, which may avoid the potential complications of pedicle avulsion and shoulder immobilization, which are known to be associated with the use of TDVs [8]. However, using IMVs has several shortcomings, such as the requirement for excision of costal cartilage and/or intercostal muscles that normally protect these vessels. Recent studies have demonstrated several complications associated with the use of IMVs, including medial contour deformities, pneumothorax, and postoperative chest pain $[9,10]$. The latter two complications can limit deep breathing and potentially increase the risk of postoperative pulmonary complications $[9,10]$. To diminish the risk of postoperative pulmonary complications, the transversus thoracic muscle in the third and fourth intercostal space may be used for coverage between IMVs and the parietal pleura [11].

We hypothesized that there would be statistically significant differences between the two recipient vessels. However, in this matched cohort study, no significant differences were found in patient demographics or clinical outcomes, including the complication rates and aesthetic outcomes. Although three variables showed significant differences in breast cancer staging, the selection of recipient vessels might not influence the complication rate or aesthetic outcome. We generally used TDVs as recipient vessels until 2010 because more advanced breast cancer patients underwent axillary lymphadenectomy or lymph node biopsy by a general surgeon. Therefore, immediate MS-TRAM flaps used TDVs as recipient vessels. In addition, TDVs were used for recipient vessels for flaps that had a sufficiently long donor pedicle, whereas IMVs were used for recipient vessels when the length of the donor pedicle was relatively short. Recently, we have preferred using IMVs for recipient vessels in accordance 
with recent trends in the field. We also consider the time required to dissect and expose the recipient vessels in immediate breast reconstructions. In addition, the mastectomy type, preexisting coronary artery disease that could require a bypass procedure, a previous operative history of breast surgery, and lymph node dissection are factors that influence the selection of recipient vessels in MS-TRAM flaps.

Several reports have compared the complication rates according to the recipient vessels used for autologous breast reconstruction $[5,12,13]$. No significant difference was found between the use of either vessel in terms of total or partial flap loss [13]. In addition, the overall incidence of complications in the IMVs was from 5\% to $12 \%$, while that in the TDVs was from $3.4 \%$ to $12 \%$ [13]. The results of this one-to-one matched study on complication rates concur with those of previous clinical and systematic studies. Saint-Cyr et al. [5] also reported that the complication rates were similar between the two groups, although the rate of seroma formation was significantly higher when TDVs were used as recipient vessels. Kropf et al. [14] reported a higher rate of fat necrosis in the TDV group.

The diameter of the recipient vessels is one of the major factors that determines the success of autologous breast reconstruction. Several cadaveric studies have reported the diameter of TDVs and IMVs and their perforators [15,16]. A cadaveric study demonstrated that the median diameter of the thoracodorsal artery was $1.5 \mathrm{~mm}$, and those of the thoracodorsal vein was $2.5 \mathrm{~mm}$. In addition, the median diameter of the internal mammary artery was $2.5 \mathrm{~mm}$, whereas that of the internal mammary vein was $3.0 \mathrm{~mm}$. In this cadaveric study, IMVs showed better match to DIEVs as recipient vessels than TDVs [15].

When considering TDVs as recipient vessels for anastomosis, a sufficient length of the pedicle should be ensured to avoid placing the TRAM flap too laterally toward the axilla. In particular, the lateral positioning of the pedicle and anastomoses away from the direct weight of the flap are important considerations in reconstruction of patients with large breast volume [17]. The use of TDVs may reduce the likelihood of a salvage latissimus dorsi musculocutaneous flap in cases of flap failure [18]. Therefore, the serratus branch of TDVs should remain connected to the distal pedicle of the latissimus dorsi muscle. Injury to the serratus branch during dissection should be avoided. Meticulous dissection is required to decrease the risk of lymphedema and nerve and shoulder injury. Anatomically, axillary lymph nodes are located adjacent to the thoracodorsal neurovascular bundle [19]. Although the incidence of lymphedema has decreased from $20 \%$ to as low as $6 \%$ since the advent of innovations in the treatment of metastatic cancer and sentinel lymph node dissection [20], meticulous and non-extensive dissection of TDVs is needed. After anastomosis, additional sutures for flap orientation are required during flap positioning and inset. In general, a narrow ptotic breast should be reconstructed with an ipsilateral flap that has been rotated $90^{\circ}$ with the umbilicus positioned in an inferomedial orientation. As a result, the height of the designed flap thus corresponds to the width of the base of the breast mount. To increase projection, the base may be folded over upon itself. A less ptotic breast or a wide breast is reconstructed with a contralateral flap rotated by about $140^{\circ}$, such that the lateral aspect of the initial flap design becomes the tail of the neo-breast mound [21].

Certain considerations must also be kept in mind for the use of IMVs. First of all, careful incision of the posterior perichondrium is required to minimize bleeding under loupe magnification. Osseous segments of the rib should not be removed during removal of costal cartilage. After anastomosis, partial repositioning of costal cartilage or repair of the perichondrium may help decrease postoperative pain or chest depression. Second, vertical movement with respiration and the concomitant transitory loss of microscope focus are pitfalls. Therefore, retaining sufficient space for anastomosis after careful dissection of IMVs is an important factor that contributes to the success of anastomosis. When a diameter mismatch between IMVs and DIEVs is expected, IMV perforators or IMVs at the level of the 4th or 5th ribs may be alternative choices of recipient vessels, although most IMVs at the level of the 2nd or 3rd ribs show a better match to DIEVs, discussed above. After anastomosis, identification of bleeding around the anastomosis sites and thorough cauterization or clipping are important because leakage of blood may result in hematoma, which is a potentially disastrous complication associated with flap congestion or loss. The final consideration is that the cutaneous incisions should be minimized. After anastomosis, the vascular pedicle should be gently redraped over the chest wall without any kinks or folds [22]. When the flap is inset using IMVs, the exact opposite of the TDV procedure should be performed. For example, a contralateral flap should be used for a narrow, pendulous breast whereas an ipsilateral flap should be used to make a wider breast mound [21].

Over the last decade, preferences for recipient vessels for autologous breast reconstruction have changed from TDVs to IMVs. IMVs have several advantages, including more stable microsurgical results, greater effectiveness in placing volume in the medial pole of the breast, and simplicity of the surgical procedure [23]. In addition, reconstructive surgeons have recently begun to prefer selecting IMV perforators as alternative recipient vessels. IMV perforators branch off from the laterodorsal border of the sternum, pass through the intercostal space, perfo- 
rate the pectoralis major muscle at its medial border, and finally perforate the overlying fascia [24]. We also reported a clinical anatomic study of IMV perforators as recipient vessels for autologous breast reconstruction [25]. Although the operation time may be reduced since the perforators can be dissected without excising of the pectoralis major muscle or costal cartilage, and complications related to chest deformities and pain are reduced, the anatomical locations of the perforators are considered less reliable, the perforators have smaller diameters, and the vessel walls are thinner and more vulnerable than those of TDVs and IMVs. Recently, we have used IMVs as recipient vessels in autologous breast reconstruction, while retaining IMV perforators as a secondary choice of recipient vessels.

Our study had all the limitations that are inherent to retrospective studies. For example, patient compliance was not considered, although it could have affected the outcomes. In addition, selection bias could have been present, as the patients analyzed in this study were mostly Koreans who underwent reconstruction at a single tertiary referral center for autologous breast reconstruction. Furthermore, this study is limited by its small sample size. Therefore, we used a one-to-one matching design to facilitate a better comparison than other comparative studies of recipient vessels for autologous breast reconstruction. Further studies with larger sample size may be required.

Despite those limitations, this study demonstrated that both TDVs and IMVs are reliable and accessible recipient vessels for autologous breast reconstruction. Nevertheless, further anatomic and large clinical studies on these recipient vessels are needed to identify the optimal choice between TDVs and IMVs. In conclusion, this study demonstrated that both TDVs and IMVs are safe and efficient as recipient vessels in terms of the complication rate and aesthetic outcomes.

\section{NOTES}

\section{Conflict of interest}

No potential conflict of interest relevant to this article was reported.

\section{Ethical approval}

The study was approved by the Institutional Review Board of Korea University Guro Hospital (IRB No. 2019GR0067) and performed in accordance with the principles of the Declaration of Helsinki. Written informed consents were obtained.

\section{Patient consent}

The patients provided written informed consent for the publication and the use of their images.

\section{Author contribution}

Study concept and design: Yoon ES. Data acquisition: Jang SY. Data analysis and interpretation: Moon KC. Drafting of the manuscript: Moon KC. Critical revision of the manuscript for important intellectual content: Lee JM. Statistical analysis: Baek SO. Administrative, technical, or material support: Lee BI, Park SH. Study supervision: Yoon ES. Approval of final manuscript: all authors.

\section{ORCID}

Kyung-Chul Moon https://orcid.org/0000-0001-6072-261X Jae-Min Lee https://orcid.org/0000-0001-6173-0611

Si-Ook Baek https://orcid.org/0000-0003-3896-5572

Seo-Yoon Jang https://orcid.org/0000-0001-7812-233X

Eul-Sik Yoon https://orcid.org/0000-0001-5734-6625

Byung-Il Lee https://orcid.org/0000-0001-8183-0388

Seung-Ha Park https://orcid.org/0000-0002-2667-2791

\section{Supplementary material}

Supplemental Video 1. After anastomosis of a thoracodorsal vessel.

Supplemental data can be found at:

https://doi.org/10.5999/aps.2018.00913.v001

Supplemental Video 2. Dissection of an internal mammary vessel.

Supplemental data can be found at:

https://doi.org/10.5999/aps.2018.00913.v002

\section{REFERENCES}

1. Chang DW, Kim S. Breast reconstruction and lymphedema. Plast Reconstr Surg 2010;125:19-23.

2. Temple CL, Strom EA, Youssef A, et al. Choice of recipient vessels in delayed TRAM flap breast reconstruction after radiotherapy. Plast Reconstr Surg 2005;115:105-13.

3. Loiselle F, Schrag C, Magi E, et al. Occult malignancy rate associated with thoracodorsal vessel dissection for free flap breast reconstruction. J Surg Oncol 2008;98:94-6.

4. Halim AS, Alwi AA. Internal mammary perforators as recipient vessels for deep inferior epigastric perforator and muscle-sparing free transverse rectus abdominis musculocutaneous flap breast reconstruction in an Asian population. Ann Plast Surg 2014;73:170-3.

5. Saint-Cyr M, Youssef A, Bae HW, et al. Changing trends in recipient vessel selection for microvascular autologous breast reconstruction: an analysis of 1483 consecutive cases. Plast Reconstr Surg 2007;119:1993-2000.

6. Moran SL, Nava G, Behnam AB, et al. An outcome analysis 
comparing the thoracodorsal and internal mammary vessels as recipient sites for microvascular breast reconstruction: a prospective study of 100 patients. Plast Reconstr Surg 2003; 111:1876-82.

7. Haywood RM, Raurell A, Perks AG, et al. Autologous free tissue breast reconstruction using the internal mammary perforators as recipient vessels. Br J Plast Surg 2003;56:68991.

8. Follmar KE, Prucz RB, Manahan MA, et al. Internal mammary intercostal perforators instead of the true internal mammary vessels as the recipient vessels for breast reconstruction. Plast Reconstr Surg 2011;127:34-40.

9. Majumder S, Batchelor AG. Internal mammary vessels as recipients for free TRAM breast reconstruction: aesthetic and functional considerations. Br J Plast Surg 1999;52:286-9.

10. Dupin CL, Allen RJ, Glass CA, et al. The internal mammary artery and vein as a recipient site for free-flap breast reconstruction: a report of 110 consecutive cases. Plast Reconstr Surg 1996;98:685-9.

11. Hefel L, Schwabegger A, Ninkovic M, et al. Internal mammary vessels: anatomical and clinical considerations. $\mathrm{Br} \mathrm{J}$ Plast Surg 1995;48:527-32.

12. Santanelli Di Pompeo F, Longo B, Sorotos M, et al. The axillary versus internal mammary recipient vessel sites for breast reconstruction with diep flaps: a retrospective study of 256 consecutive cases. Microsurgery 2015;35:34-8.

13. Samargandi OA, Winter J, Corkum JP, et al. Comparing the thoracodorsal and internal mammary vessels as recipients for microsurgical autologous breast reconstruction: a systematic review and meta-analysis. Microsurgery 2017;37: 937-46.

14. Kropf N, Macadam SA, McCarthy C, et al. Influence of the recipient vessel on fat necrosis after breast reconstruction with a free transverse rectus abdominis myocutaneous flap. Scand J Plast Reconstr Surg Hand Surg 2010;44:96-101.
15. Lhuaire M, Hivelin M, Drame M, et al. Determining the best recipient vessel site for autologous microsurgical breast reconstruction with DIEP flaps: an anatomical study. J Plast Reconstr Aesthet Surg 2017;70:781-91.

16. Rosson GD, Holton LH, Silverman RP, et al. Internal mammary perforators: a cadaver study. J Reconstr Microsurg 2005;21:239-42.

17. Banwell M, Trotter D, Ramakrishnan V. The thoracodorsal artery and vein as recipient vessels for microsurgical breast reconstruction. Ann Plast Surg 2012;68:542-3.

18. Santanelli F, Longo B, Cagli B, et al. Predictive and protective factors for partial necrosis in DIEP flap breast reconstruction: does nulliparity bias flap viability? Ann Plast Surg 2015; 74:47-51.

19. Cody 3rd HS. Sentinal lymph node mapping in breast cancer. Breast Cancer 1999;6:13-22.

20. McLaughlin SA, Wright MJ, Morris KT, et al. Prevalence of lymphedema in women with breast cancer 5 years after sentinel lymph node biopsy or axillary dissection: objective measurements. J Clin Oncol 2008;26:5213-9.

21. Neligan P, Warren RJ, Van Beek A. Plastic surgery. 3rd ed. New York: Elsevier Saunders; 2013.

22. Nahabedian $M$. The internal mammary artery and vein as recipient vessels for microvascular breast reconstruction. Ann Plast Surg 2012;68:537-8.

23. Yang SJ, Eom JS, Lee TJ, et al. Recipient vessel selection in immediate breast reconstruction with free abdominal tissue transfer after nipple-sparing mastectomy. Arch Plast Surg 2012;39:216-21.

24. Schmidt M, Aszmann OC, Beck H, et al. The anatomic basis of the internal mammary artery perforator flap: a cadaver study. J Plast Reconstr Aesthet Surg 2010;63:191-6.

25. Baek IS, You JP, Rhee SM, et al. A clinical anatomic study of internal mammary perforators as recipient vessels for breast reconstruction. Arch Plast Surg 2013;40:761-5. 